\title{
Vitamin D status and muscular side-effects in statin users: preliminary findings from a seasonal observation study
}

\author{
E.L. Carson ${ }^{1}$, L.K. Pourshahidi ${ }^{1}$, M.J. O'Kane ${ }^{2}$, J.J. Strain ${ }^{1}$ and M.S. Mulhern ${ }^{1}$ \\ ${ }^{1}$ Northern Ireland Centre for Food and Health (NICHE), University of Ulster, Coleraine, Northern Ireland, BT52 ISA \\ and ${ }^{2}$ Department of Clinical Chemistry, Altnagelvin Area Hospital, Londonderry, Northern Ireland, BT47 6SB
}

Approximately 7 million people in the UK are currently taking statin medication to lower cholesterol and prevent cardiovascular events $^{(1)}$. Adverse muscular side-effects of statins are a common cause of treatment discontinuation. Low vitamin D status has been associated with a higher prevalence of statin-associated myalgia, the most common muscular side-effect and normalising vitamin $\mathrm{D}$ status has been shown to resolve symptoms ${ }^{(2)}$. This study aimed to investigate seasonal changes in vitamin $\mathrm{D}$ status and any associated changes in muscular side-effects in statin users.

To date, 26 stable statin users (same type and dose $\geqslant 3$ months, 10 male and 16 female) have completed both study visits; once at the end of summer (peak of vitamin D status) and once at the end of winter (nadir of vitamin D status). These participants had genotypically proven heterozygous familial hypercholesterolaemia $(\mathrm{FH})$ and had not been diagnosed with any muscular conditions or complications. At each visit, fasting blood samples were collected and analysed for serum 25 hydroxyvitamin $\mathrm{D}(25(\mathrm{OH}) \mathrm{D}) \mathrm{concen}-$ tration using the gold-standard, liquid chromatography tandem-mass spectrometry (LC-MS/MS; API4000, AB SCIEX). Height (cm) was taken once and weight $(\mathrm{kg})$ and fat free mass (FFM, kg) were measured at both time-points, using bioelectrical impedance analysis (Tanita Cooperation, Tokyo, Japan). Grip strength (GS; kg) was measured in triplicate by hand grip dynamometry (Takei Scientific Instrument Company Limited, Japan) and muscular pain was quantified using the validated McGill pain questionnaire ${ }^{(3)}$ at each seasonal time-point.

\begin{tabular}{|c|c|c|c|c|c|}
\hline & \multicolumn{2}{|c|}{ End of summer } & \multicolumn{2}{|c|}{ End of winter } & \multirow[t]{2}{*}{$P$} \\
\hline & Median & $25^{\text {th }}, 75^{\text {th }}$ centile & Median & $25^{\text {th }}, 75^{\text {th }}$ centile & \\
\hline $25(\mathrm{OH}) \mathrm{D}(\mathrm{nmol} / \mathrm{L})$ & $41 \cdot 5$ & $31 \cdot 2,76 \cdot 5$ & $27 \cdot 7$ & $21 \cdot 0,56 \cdot 8$ & $<0.001 *$ \\
\hline BMI $\left(\mathrm{kg} / \mathrm{m}^{2}\right)$ & $29 \cdot 8$ & $25 \cdot 8,32 \cdot 4$ & $30 \cdot 2$ & $25 \cdot 7,32 \cdot 3$ & 0.663 \\
\hline FFM (kg) & $49 \cdot 7$ & $44 \cdot 3,62 \cdot 2$ & $49 \cdot 9$ & $43.4,61.8$ & $0 \cdot 087$ \\
\hline$\%$ FFM & $64 \cdot 1$ & $60 \cdot 1,71 \cdot 3$ & $62 \cdot 4$ & $58.4,69 \cdot 8$ & $0 \cdot 188$ \\
\hline FFMI $\left(\mathrm{kg} / \mathrm{m}^{2}\right)$ & $18 \cdot 7$ & $17 \cdot 9,20 \cdot 3$ & $19 \cdot 0$ & $17 \cdot 4,20 \cdot 1$ & 0.087 \\
\hline GS (kg) & $24 \cdot 0$ & $20 \cdot 2,46 \cdot 0$ & $25 \cdot 0$ & $22 \cdot 5,43 \cdot 5$ & 0.97 \\
\hline McGill Pain score $(\%)$ & $53 \cdot 2$ & $47 \cdot 7,57 \cdot 0$ & $51 \cdot 9$ & $45 \cdot 8,56 \cdot 1$ & $0 \cdot 648$ \\
\hline
\end{tabular}

Paired samples $t$ test. *Significance at $P<0.05$. (BMI, body mass index; FFM, fat free mass; FFMI, fat free mass index [fat free mass corrected for height]; GS, grip strength).

This group consisted of atorvastatin ( $n$ 15), rosuvastatin $(n$ 10) and simvastatin $(n$ 1) users, with a mean (SD) age of 52.4 (14.1) years. Muscle pain was reported by $42 \%$ of the group at the end of summer and $31 \%$ at the end of winter. End of summer 25 $(\mathrm{OH}) \mathrm{D}$ concentration was significantly higher than at the end of winter; however, muscular outcome measures (FFM, GS or muscle pain frequencies/scores) did not differ significantly between these time-points. Correlation analyses also suggest no association between 25(OH)D concentration and FFM, GS or muscle pain scores in the overall group, or when split by statin-type.

Muscular side-effects of statin use do not appear to be associated with seasonal changes in vitamin D status; however, this is preliminary analysis on half of the overall sample size originally calculated to power this study $(n 50)$. It is noteworthy that muscle pain was self-reported and future studies should consider other biochemical measures, such as creatine kinase, as a measure of muscle damage.

This work was funded by the Department for Employment and Learning \& WHSCT/BMSRI matched funding. Ethical approval was obtained from the Office of Research Ethics Committee Northern Ireland (ORECNI) and the study was conducted according to the guidelines laid down in the Declaration of Helsinki.

1. NICE (2014) NICE clinical guideline CG181. Available at www.nice.org/guidance/cg181

2. Ahmed et al. (2009) Translation. Res. 153, 11-16.

3. Melzack (1975) Pain 1, 277-299. 